\title{
Revisión y validación de la evapotranspiración potencial (ETP) media anual y media mensual en Honduras obtenida por métodos ambientales de percepción remota
}

\author{
Lenín Henríquez Dole y Anahí J. Miner Vega ${ }^{1}$
}

\section{RESUMEN}

La evapotranspiración (ET) es un proceso esencial en el ciclo hidrológico del planeta y es de mucha importancia para el balance hídrico, especialmente en el riego. La evapotranspiración potencial (ETP) es la medida de la máxima de ET posible en condiciones óptimas, su estimación es muy compleja y para ello se han planteado ecuaciones empíricas en diversas partes del mundo. En Honduras se han realizado escasos trabajos de estimación de la ETP utilizando estas metodologías, aplicándose por lo general las que menos datos requieren.

Se verificó mediante test estadísticos que, aunque existen mediciones a nivel nacional, la temperatura media no es un dato confiable en la mayoría de las series de las estaciones meteorológicas del país, lo que complica aún más el cálculo de ETP. Recientemente se ha publicado, como alternativa a la estimación empírica de la ETP, el mapa global de evapotranspiración potencial (Global-PET), que se basa en el método de Hargreaves para estimar la ETP, generado por el Consortium for Spatial Information (CGIAR-CSI).

Se realizaron análisis estadísticos comparando los datos de la ETP media mensual y anual estimada por el método de Hargreaves con los valores aportados por el Global-PET y se concluye que representa correctamente la variabilidad, excepto en el mes de septiembre. Se encontró también que existe un gradiente (-32 $\mathrm{mm}$ por cada $100 \mathrm{~m}$ de altura) entre la ETP estimada por este método y las elevaciones mayores a $150 \mathrm{msnm}$. Los mapas de Global-PET representan una nueva base de datos confiable, revisada y validada a nivel nacional, sin costo alguno y accesible en internet para poder hacer uso de ella con fines no comerciales.

Palabras clave: Evapotranspiración potencial, percepción remota, temperatura, validación de base de datos, Honduras.

\footnotetext{
${ }^{1}$ Universidad Nacional Autónoma de Honduras. Facultad de Ciencias, Instituto Hondureño de Ciencias de la Tierra.Correo electrónico: henriquez.lenin@gmail.com; anahiminer@gmail.com
} 


\section{ABSTRACT}

Evapotranspiration (ET) is an essential process in the planet's hydrologic cycle, and a very important estimation for water balance, especially in irrigation. Potential Evapotranspiration (PET) is the measure of the maximum ET possible in optimal conditions, its assessment is complex and empirical equations all over the world have been done to this purpose. In Honduras few works have been done about PET's estimation using empirical methodologies, applying usually the one with less data requirements. Using double mass and statistical test analysis have been verified that average temperature is not a reliable record in most of the meteorological stations in the country.

For that reason, the PET estimation is more complicated. Recently, an alternative to empirical estimation has been published: Global Potential Evapotranspiration Map (Global-PET) based on Hargreaves method to PET's evaluation, generated by Consortium for Spatial Information (CGIAR-CSI).

It has been statistically proved that Global-PET represents PET's annual and monthly variability using Hargreaves method, except September. There is also a gradient (-32 mm per each 100 meters of elevation) between PET and elevations above $150 \mathrm{~m}$. Global-PET maps represent a new reliable, checked and validated database at national level, without any cost and accessible on internet for those who may want to use it for non commercial purpose.

Keywords: Potential Evapotranspiration, Remote Sensing, Temperature, Database Validation, Honduras.

\section{INTRODUCCIÓN}

La evapotranspiración potencial (ETP) es un proceso esencial en el ciclo hidrológico del planeta. Es una medida de la máxima cantidad de agua que puede ser transferida desde la superficie de la tierra, cubierta de vegetación, a la atmósfera y cuya estimación solo depende de las condiciones climáticas en el momento del cálculo (Thornthwaite, 1948).

A fines de los años setenta y comienzos de los años ochenta del siglo pasado, la FAO hace más precisa la estimación de este proceso e introduce el concepto de evapotranspiración del cultivo de referencia (ETo) como una mejora a la estimación de este flujo. Así, la ETo se define como la suma de evaporación y transpiración en un cultivo de referencia bajo condiciones óptimas de riego, asumiendo una altura de 
12 centímetros, una resistencia de la superficie de $70 \mathrm{~s} / \mathrm{m}$ y un albedo de 0.23 , lo que se asemeja a la evaporación que ocurre en una superficie extensa de pasto verde de altura uniforme, creciendo activamente y sin déficit de agua (Allen, 1998).

En la actualidad, el término evapotranspiración del cultivo de referencia ha sido reemplazado por el de evapotranspiración de referencia. No obstante, aunque se reconoce que los conceptos de ETP y ETo, en estricto sentido, son diferentes, ambos indican cantidades máximas de agua perdida a favor de la atmósfera. Por ello en algunos estudios, incluyendo este, han sido considerados equivalentes (McKenney y Rosenberg, 1993).

Se estima como promedio global que el $57 \%$ de la precipitación anual es transferida a la atmósfera a través de este proceso, pudiendo llegar a valores del $90 \%$ y $100 \%$ en zonas áridas o desérticas, respectivamente (Sánchez, 1992). La ETo es un parámetro de interés en la clasificación climática de los diferentes ambientes. Además, facilita una adecuada gestión de los recursos hídricos y económicos necesarios para la construcción de obras y planificación del riego, ya que permite la definición de periodos de déficit o exceso hídrico, entendida esta como la demanda de agua que tienen los diferentes cultivos y plantaciones (Valenzuela y Ferreira, 1985; Hargreaves, 1994; Comisión Nacional de Riego, 1997; Xu y Singh, 2001; Droogers y Allen, 2002; Temesgen, 2005; López-Moreno, 2009).

La intensidad de este flujo de agua entre el suelo y la atmósfera se ve determinada por diversas variables como cobertura vegetal, tipo de suelo, uso del suelo, tipo de vegetación, densidad de la vegetación, tiempo de vida de las especies, tipos de cultivos, temperatura del aire, radiación solar, humedad relativa y velocidad del viento, entre otras. Por la complejidad de este proceso y del cual la transpiración de las plantas forma una parte muy importante, se han propuesto metodologías entre las que se encuentran expresiones empíricas basadas en observaciones puntuales en diversas partes del planeta (Thornthwaite, 1948; Hargreaves, 2003; Allen, 1998).

Aunque la aplicación de estas metodologías se puede intuir a nivel mundial, aún necesitan ser validadas en otras regiones lejos de aquellas donde fueron deducidas. La más ampliamente utilizada es la fórmula de Penman-Monteith, considerada como método estándar, físicamente basado, que puede ser utilizado globalmente. Sin embargo, esta fórmula tiene la desventaja de requerir una gran cantidad de parámetros específicos que muchas veces no son medidos en estaciones meteorológicas comunes, incrementándose esta deficiencia en países en desarrollo (Droogers y Allen, 2002). 
En Honduras se han realizado escasos trabajos de estimación de la ETP, siendo uno de estos el realizado por Jiménez y Hernández (1990), quienes calcularon la ETP con varias metodologías e hicieron análisis de regresión lineal para llegar a estimaciones de la ecuación de Penman utilizando otros métodos. Otra estimación de ETP a nivel nacional fue la realizada por CEDEX en el balance hídrico de 2002, quienes utilizaron la fórmula de Hargreaves corrigiendo los resultados mediante un coeficiente para obtener la evapotranspiración de referencia de Penman-Monteith. La Secretaría de Recursos Naturales y Ambiente (SERNA) también ha realizado un mapa de evapotranspiración potencial publicado en su web. De igual manera, se han realizado trabajos a nivel de cuenca o microcuenca que presentan resultados puntuales para las zonas de estudio.

A nivel mundial, el Consortium for Spatial Information (CGIAR-CSI) con el apoyo del International Center for Tropical Agriculture (CIAT), ha publicado una base de datos en formato raster: el mapa global de evapotranspiración potencial (Global-PET) para uso no comercial. Esta información pretende contribuir con estudios sobre desarrollo sostenible, biodiversidad y conservación, disminución de la pobreza y adaptación a los procesos de cambio climático, en particular en países en vías de desarrollo.

El presente estudio pretende revisar y validar el mapa de la ETP Global (Global$\mathrm{PET}$ ), de $1 \mathrm{~km}$ de resolución, en Honduras, comparándolo con estimaciones puntuales de la ETP, obtenidas a partir de las bases de datos climáticos hondureños, a nivel mensual y anual. Se pretende lograr una nueva fuente de información, de acceso gratuito y de fácil manejo que proporcione una referencia de la ETP en cualquier lugar de Honduras.

\section{MATERIALESYMÉTODOS}

\section{Base de datos}

La base de datos utilizada ha sido compuesta por los datos mensuales provenientes de estaciones meteorológicas gestionadas por la SERNA, la Empresa Nacional de Energía Eléctrica (ENEE), el Servicio Meteorológico Nacional (SMN), el Servicio Autónomo Nacional de Acueductos y Alcantarillado (SANAA), la Fundación Hondureña de Investigación Agrícola (FHIA) y otras instituciones privadas. 
Cuadro 1. Número de estaciones que miden temperatura dentro de la base de datos recopilada

\begin{tabular}{|l|c|}
\hline \multicolumn{1}{|c|}{ Parámetro } & $\begin{array}{c}\text { Número de } \\
\text { estaciones }\end{array}$ \\
\hline Temperatura media mensual & 79 \\
\hline Temperatura máxima promedio mensual & 75 \\
\hline Temperatura mínima promedio mensual & 74 \\
\hline
\end{tabular}

Fuente: Elaboración propia.

Dentro de la base de datos recopilada se separaron aquellas estaciones que medían la temperatura del aire (ver cuadro 1). Se rellenaron las series de datos de las estaciones que no disponían de valores actualizados. La calidad de los datos se comprobó mediante un test propuesto por Menne (2009), en el cual se verifica que los valores contenidos en una serie no se alejen de la media más de 3.5 veces el valor de la desviación estándar. En el caso de que algún valor superara este umbral, se marcó como dudoso. De estas estaciones se seleccionaron finalmente 48 que poseían el dato de la temperatura media, temperatura máxima y temperatura mínima (ver mapa 1).

Mapa 1. Ubicación de las 48 estaciones seleccionadas para el estudio de la ETP en Honduras

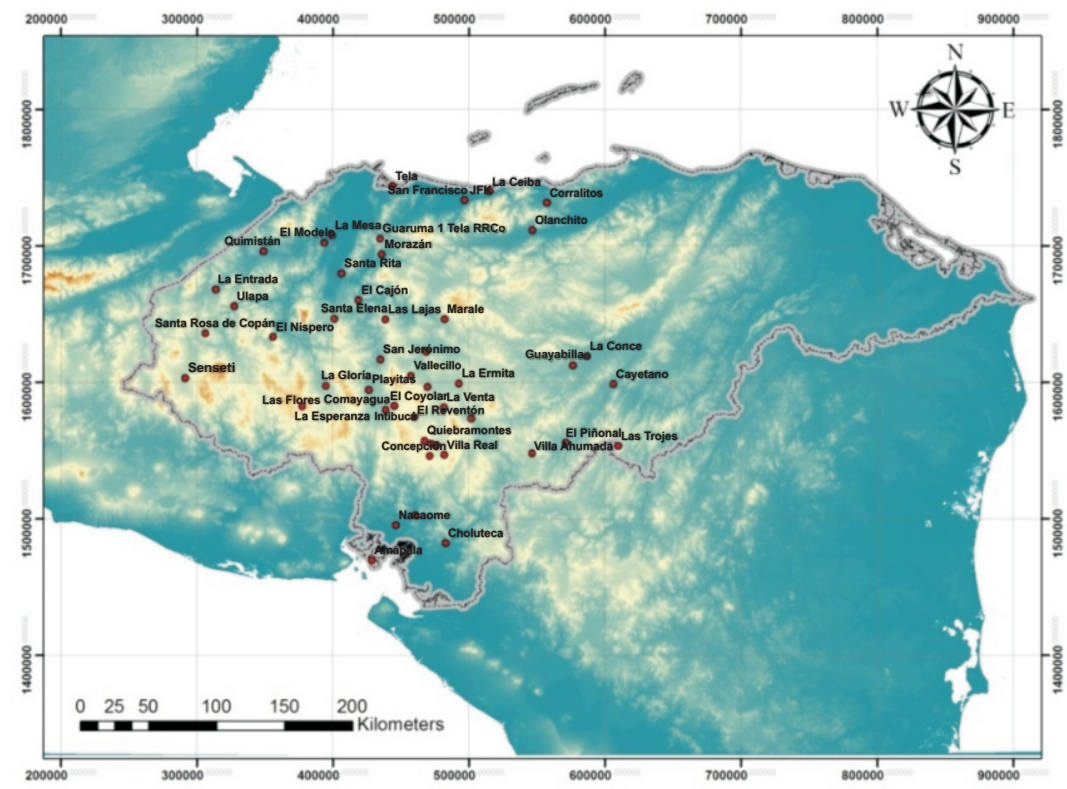

Fuente: Elaboración propia, utilizando la información brindada por las instituciones mencionadas. 
Considerando los vacíos de información y que muchas de las estaciones fueron instaladas a principios de los años 70 , se eligió un periodo común de estudio entre 1975 y 2005, con el fin de completar 30 años de datos mensuales continuos, ya que estadísticamente se considera una cantidad de años representativa (Sokolov y Chapman, 1981).

Para el relleno de datos faltantes se ha utilizado una regresión estocástica múltiple bivariada, procedimiento incluido como un módulo del software: Cálculo hidrometeorológico de aportaciones y crecidas (CHAC). Con esta metodología, el dato faltante se obtiene a partir de otras dos estaciones que, dentro de un grupo de estaciones cercanas, tienen mejor correlación (CEDEX, 2013).

Mapa 2. Global-PET: ETP anual en la zona de Honduras y alrededores

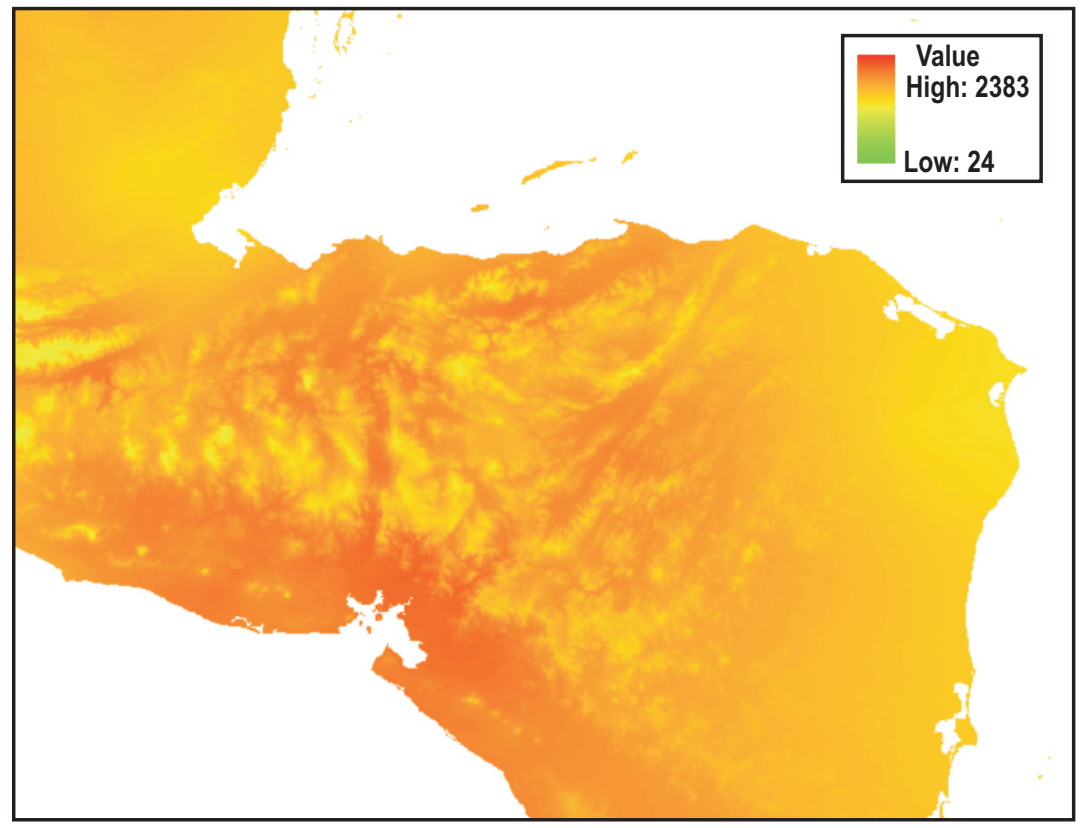

Fuente: Consortium for Spatial Information (CGIAR-CSI).

\section{Mapa Global-PET}

El mapa Global-PET generado por CGIAR-CSI ${ }^{2}$ está disponible en formato de grilla geoespacial (ARC/INFO) estándar para ESRI, siendo su resolución de 30 segundos de arco 0 , aproximadamente, $1 \mathrm{~km}$ en el Ecuador.

${ }^{2}$ Al respecto ver: http://www.cgiar-csi.org/data/global-aridity-and-pet-database 
Los parámetros que se utilizaron como input para el cálculo de la ETP fueron obtenidos de la base de datos de alta resolución World Clim Global Climate Data (Hijmans, 2005) de 30 segundos de arco, basada en un gran número de observaciones climáticas entre 1950 y 2000; también de datos topográficos de Shuttle Radar Topography Mission (SRTM). Los datos medios mensuales utilizados fueron precipitación, temperatura media, máxima y mínima.

La ETP media mensual ha sido caracterizada y luego estimada utilizando 4 métodos diferentes basados en la temperatura para definir su precisión: Thornthwaite (1948), Thornthwaite modificado por Holland (1978), Hargreaves (1985), Hargreaves modificado por Droogers y Allen (2002).

Los valores obtenidos por estos métodos fueron comparados con los resultados de la ecuación de Penman-Monteith (FAO-PM) estimada en estaciones climáticas de América del Sur y África. Entre los modelos elegidos, el que más se acerca (media similar y menor desviación típica) a la ETP de FAO-PM es el método de Hargreaves (utilizado para la generación del mapa), que requiere datos de radiación solar extraterrestre, temperatura media, temperatura mínima y máxima, cuya fórmula es la siguiente:

$$
E T_{0}: 0.0023 R_{a}\left(T_{\text {media }}+17.8\right) \sqrt{T D}
$$

Donde: $\mathrm{ET}_{0}$ es la evapotranspiración de referencia diaria (mm/día)

$R_{a} \quad$ es la radiación extraterrestre al tope de la atmósfera (mm/día)

$\mathrm{T}_{\text {media }}$ temperatura media diaria $\left({ }^{\circ} \mathrm{C}\right)$

TD diferencia entre la temperatura máxima y mínima diaria $\left({ }^{\circ} \mathrm{C}\right)$

\section{Cálculo de la ETPen Honduras}

Se realizaron los cálculos de ETP en las estaciones seleccionadas en Honduras mediante la misma fórmula de Hargreaves que se utilizó para la creación del mapa del CGIAR (Hargreaves, 1985). La radiación solar extraterrestre se estimó mediante ecuaciones de regresión lineal en cada mes basadas en la tabla presentada en el documento de FAO 56 (Allen, 1998) en función de la latitud y el mes. El valor de temperatura media mensual se calculó como la semisuma entre la temperatura promedio máxima mensual y la temperatura promedio mínima mensual de cada estación. Los cálculos se realizaron en una tabla de Excel, obteniendo inicialmente la ETP diaria de cada estación y posteriormente la ETP mensual, multiplicando por 30, 31 o 28 según el mes del año. La ETP anual es la suma algebraica de todos los meses. 


\section{Validación}

La comparación de los resultados obtenidos en las diferentes estaciones con el mapa de ETP creado por el CGIAR se realizó a nivel mensual y anual mediante la comparación visual de ambas series de valores, estimando el coeficiente de correlación entre los datos, el error cuadrático medio, realizando el test de t-student, el análisis de la varianza (ANOVA) y el test de bondad de ajuste de $X^{2}$.

\section{RESULTADOS}

\section{Análisis de las bases de datos}

La base de datos recopilada originalmente contenía al menos 70 estaciones que medían la temperatura, sin embargo, no todas ellas eran aptas para el estudio de la variabilidad espacial y temporal de este parámetro. Las 48 estaciones finalmente escogidas cumplieron con los siguientes criterios: poseer al menos 10 años de información continua en el período seleccionado (entre 1975 y 2005), no presentar tendencias ni valores atípicos dentro de sus series temporales y poseer registros en el mismo período de la temperatura media, temperatura máxima y la temperatura mínima (ver gráfico 1). Los criterios se aplicaron a las tres series de temperaturas medidas en cada estación: media, máxima y mínima.

Gráfico 1. Período de estudio seleccionado (1975-2005) de la temperatura media y estaciones eliminadas mediante los criterios de selección

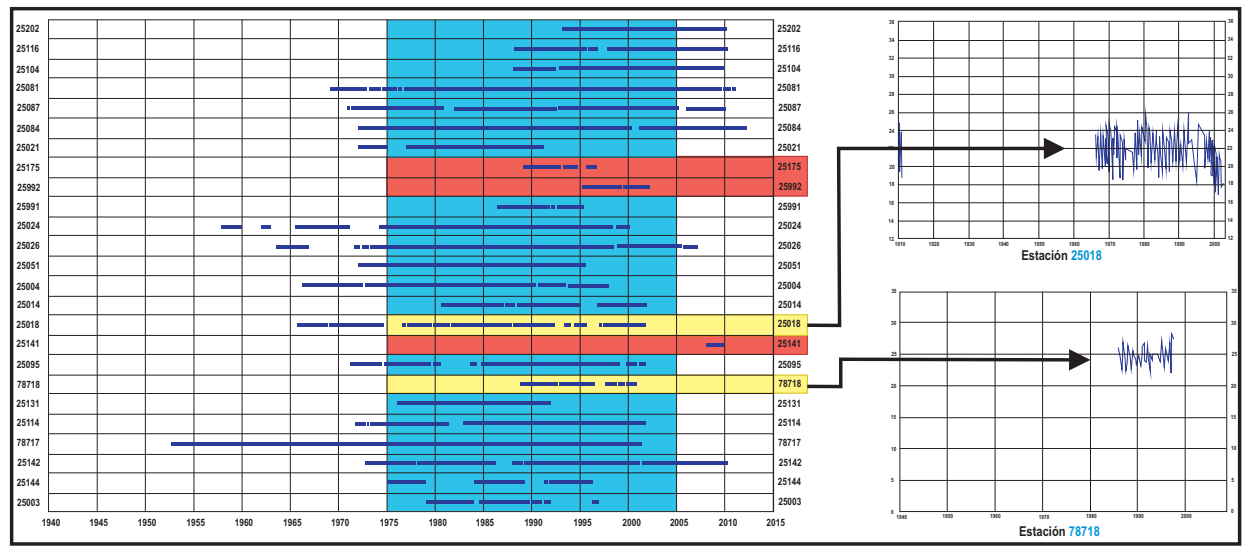

Las líneas azules representan los meses en que hay datos tomados. En el eje de las $X$ se muestra el periodo de tiempo y en el eje de las Y el código de la estación meteorológica. En rojo se presentan las series que no cumplen con la cantidad de años requerida; en amarillo se muestran series de datos con tendencias y las azules quedaron elegidas dentro de la base de datos. 
Cuadro 2 . Estaciones seleccionadas para ser parte del estudio de la evapotranspiración

\begin{tabular}{|l|l|l|l|l|l|}
\hline $\begin{array}{c}\text { Código } \\
\text { nacional }\end{array}$ & \multicolumn{1}{|c|}{ Nombre } & $\begin{array}{c}\text { Código } \\
\text { nacional }\end{array}$ & \multicolumn{1}{|c|}{ Nombre } & $\begin{array}{c}\text { Código } \\
\text { nacional }\end{array}$ & \multicolumn{1}{|c|}{ Nombre } \\
\hline 23010 & Quimistán & 25116 & Marale & 54001 & Perspire \\
\hline 23011 & La Entrada & 25131 & Sensenti & 54002 & Nacaome \\
\hline 25004 & La Gloria & 25142 & El Níspero & 56001 & La Venta \\
\hline 25014 & El Cajón & 25144 & El Modelo & 56071 & $\begin{array}{l}\text { Ingenio, El } \\
\text { Provenir }\end{array}$ \\
\hline 25024 & Las Flores, & 25202 & Las Lajas & 56106 & Concepción \\
\hline $\begin{array}{l}\text { Comayagua } \\
25026\end{array}$ & El Coyolar & 25993 & Guaruma & 56301 & El Batallón \\
\hline 25051 & Santa Clara & 27013 & San Francisco, & 56303 & Quiebramontes \\
\hline 25056 & Morazán & 31007 & Corralitos & 56602 & Villa Real \\
\hline 25080 & La Ermita & 33004 & Olanchito & 56701 & El Reventón \\
\hline 25081 & Agua Caliente, F.M. & 39001 & Cayetano & 78700 & Amapala \\
\hline 25083 & Santa Rita & 39003 & Guayabillas & 78705 & La Ceiba \\
\hline 25084 & Playitas & 39009 & Villa Ahumada & 78706 & Tela \\
\hline 25085 & Santa Elena & 39017 & El Piñonal & 78708 & La Mesa \\
\hline 25087 & San Jerónimo & 39027 & La Conce & 78717 & $\begin{array}{l}\text { Santa Rosa de } \\
\text { Copán }\end{array}$ \\
\hline 25104 & Vallecillo & 45103 & Las Trojes & 78720 & Tegucigalpa \\
\hline 25114 & Ulapa & 46999 & La Esperanza, & 78724 & Choluteca \\
\hline & & Intibucá & & \\
\hline
\end{tabular}

Basados en los criterios anteriores y agrupando las diferentes estaciones por zonas, se procedió a identificar los valores atípicos de las diferentes series. Dado que el análisis se realizó en grupos, ${ }^{3}$ se decidió disminuir el umbral de la desviación típica a 3.5, en vez de 5 como lo propone Menne (2009). El cuadro 3 muestra un ejemplo de las tablas elaboradas de identificación de valores atípicos en las 48 estaciones seleccionadas.

\footnotetext{
${ }^{3}$ La división por grupos se hizo basándose la proximidad de las estaciones, la cuenca en donde se encuentran y la relación que puede existir entre ellas, a criterio de experto. El país se ha dividido en seis (6) zonas.
} 
Cuadro 3. Valores atípicos en las series de temperatura máxima promedio mensual

\begin{tabular}{|l|l|l|}
\hline \multicolumn{1}{|c|}{ Nombre de estación } & \multicolumn{1}{|c|}{$\begin{array}{c}\text { Fecha de valor atípico } \\
\text { mensual }\end{array}$} & \multicolumn{1}{c|}{ Categoría del valor } \\
\hline San Jerónimo & Mar/abr-83 & Alto/alto \\
\hline La Ermita & Abr-72, feb-98, oct/nov-08 & alto, alto, alto/alto \\
\hline Vallecillo & Jul/ago/sep/oct-09 & Alto/alto/alto/alto \\
\hline Coyolar & Mar-01 & Alto \\
\hline Las Flores & Ene-91 & Alto \\
\hline El Cajón & May-90 & Bajo \\
\hline Santa Rosa de Copán & Feb-76, jul-84, ago-85, & Bajo, bajo, bajo, bajo, \\
\hline & bajo-89, oct-99 \\
\hline Santa Elena & Jul-79 & Bajo \\
\hline El Níspero & Abr-91, ago-03, oct-03 & Bajo \\
\hline Ulapa & May-75, ago-75 & Bajo, alto, alto \\
\hline La Ceiba & Feb-70 & alto, alto \\
\hline Villa Ahumada & May-98 & Bajo \\
\hline Guayabillas & Mar-66, abr-72 & Alto \\
\hline El Batallón & Mar-97 & Alto, alto \\
\hline Tegucigalpa & Jun/jul-50 & Bajo \\
\hline Choluteca & May-00 & Bajo/bajo \\
\hline
\end{tabular}

El análisis de los valores atípicos muestra que no existe una relación aparente entre estaciones. Es de esperarse que si existe un valor atípico o extremo, este se reproduzca en las estaciones aledañas; sin embargo, esto no se comprueba para ninguna de las fechas de los valores obtenidos. En consecuencia, se puede deducir que los valores atípicos no se producen en la región por olas de calor, sino por las inconsistencias en la medición de origen humano o instrumental.

Para detectar inconsistencias en las series de datos se realizó el análisis de doble masa, comparando diferentes estaciones con la media de los diferentes grupos escogidos. Este análisis reveló que la temperatura media mensual presentaba muchas deficiencias en sus mediciones, sin embargo, no era el caso con la temperatura máxima promedio mensual ni la temperatura mínima promedio mensual.

La razón aparente de estas inconsistencias en la temperatura media mensual responde a que esta temperatura no es una medición, sino una estimación diaria basada en las temperaturas que se toman a lo largo del día. Las inconsistencias encontradas en los datos de la temperatura media mensual han hecho que se 
procediera a descartar este parámetro y se utilizara como la temperatura media mensual, el promedio entre la temperatura máxima promedio mensual y la mínima promedio mensual, datos que sí probaron ser series consistentes a lo largo del tiempo, como se muestra en el gráfico 2.

Gráfico 2. Diagrama de doble masa de la temperatura máxima promedio mensual (arriba), la temperatura media mensual (centro) y la temperatura mínima mensual (abajo) en tres estaciones: Las Flores (25024), San Jerónimo (25087) y Choluteca (78724)

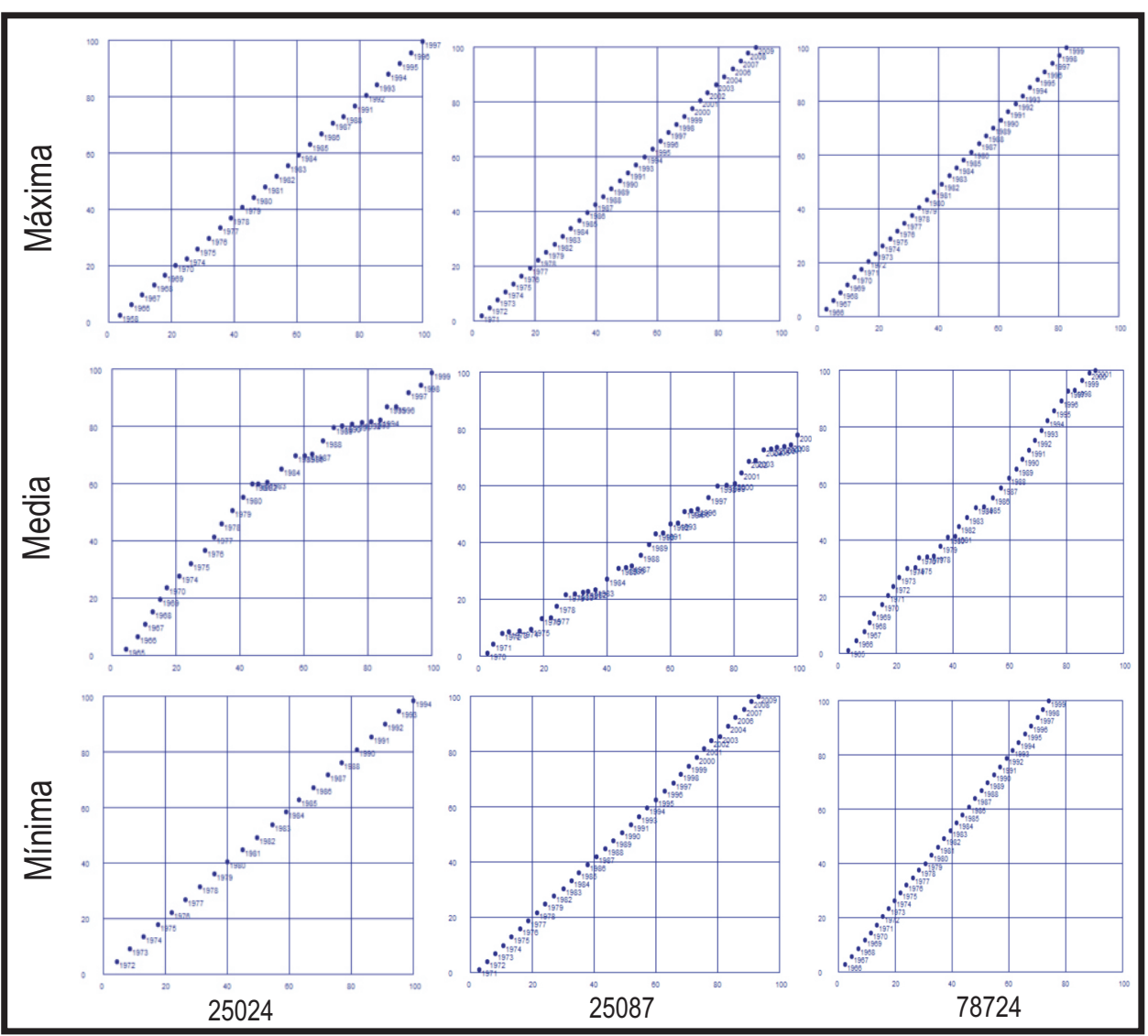

La homogeneidad y consistencia en los registros de las temperaturas máximas y mínimas promedio mensual, asegura que el cálculo de la temperatura media mensual, a partir de ellas, también sea una serie homogénea y consistente. Se recomienda que el cálculo de la temperatura media sea revisado, estandarizado y homogeneizado en todas las estaciones de Honduras. 


\section{Cálculo de la ETP en Honduras}

Hargreaves (1985) sostienen que la ETP está en función de la temperatura y la radiación solar. Si se asume que a lo largo del año la distribución de la radiación solar es invariante en el tiempo, entonces la ETP queda íntimamente ligada a la variación de la temperatura en un lugar determinado. Esto se puede comprobar en el gráfico 3, en donde la ETP calculada responde a la misma variación de la temperatura.

Gráfico 3. Distribución de la temperatura media (izquierda) y la ETP (derecha) en la estación Quimistán
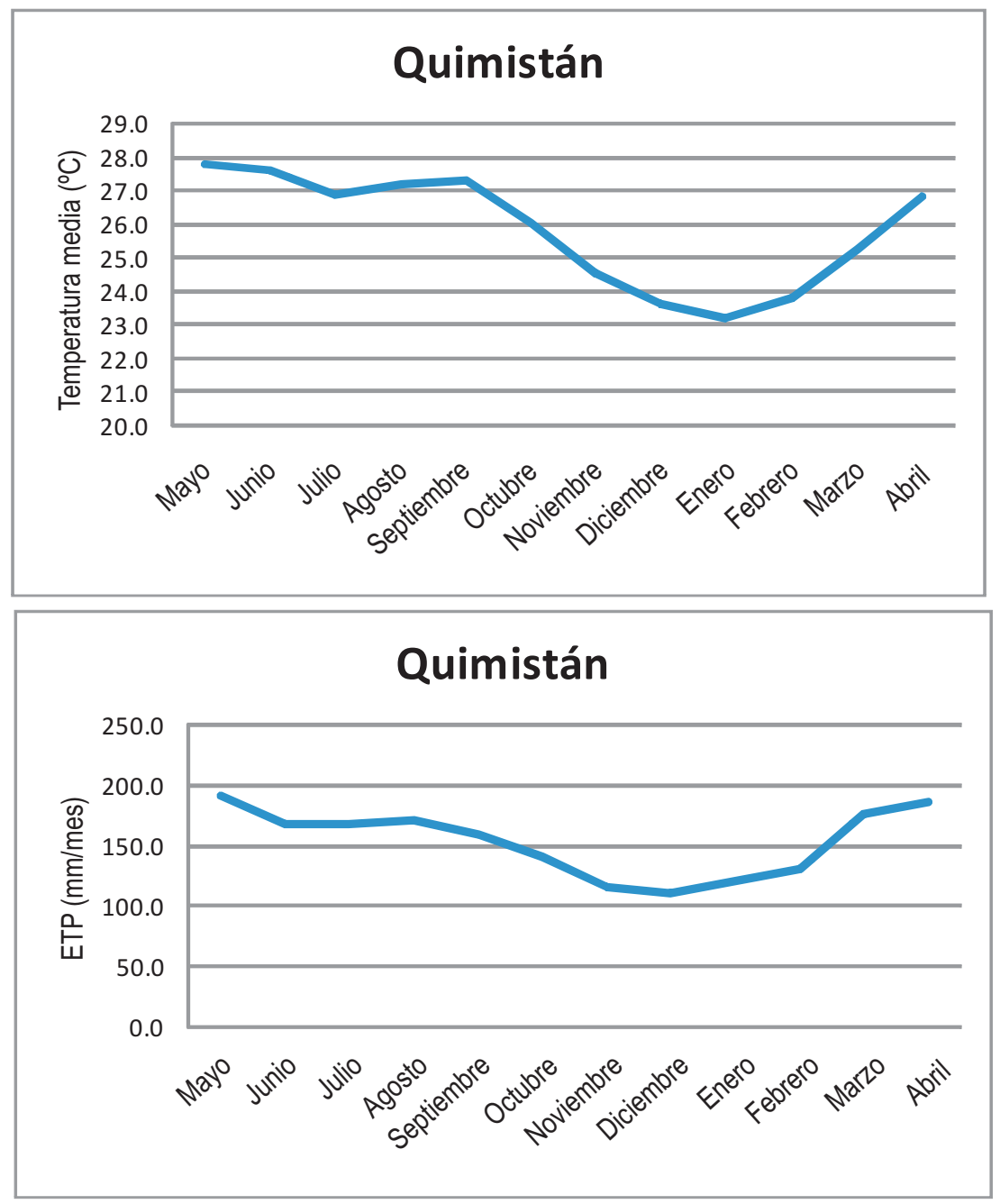


\section{Gráfico 4. Distribución de la ETP en Honduras}

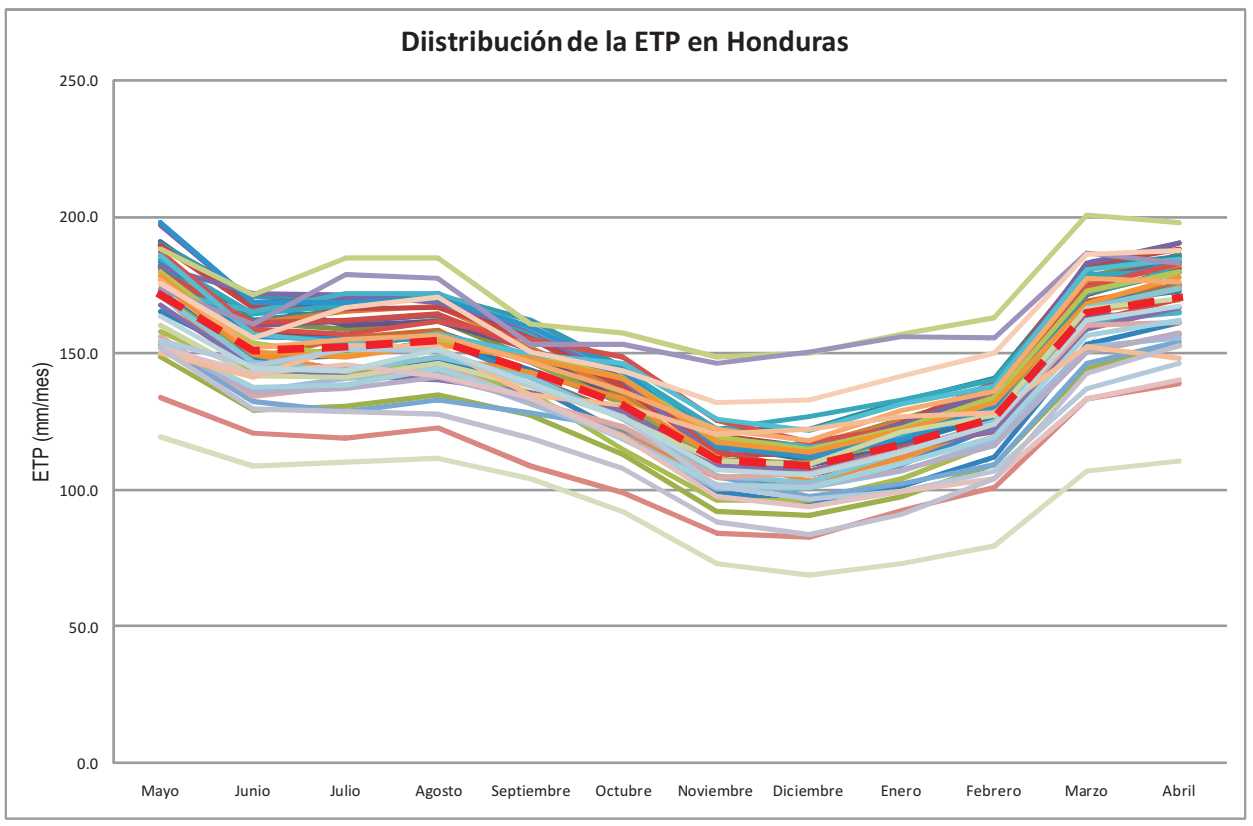

La línea roja discontinua representa la ETP media de todas las estaciones.

Se puede apreciar que tanto la distribución de la temperatura como de la ETP en el país (ver gráfico 4), tienen sus máximos en abril y mayo y sus mínimos en noviembre, diciembre y enero. Agosto representa una alteración al alza debido a la influencia del anticiclón de las Bermudas que se acerca al istmo centroamericano durante estas fechas (Zúniga, 1990).

Como promedio anual se estima que Honduras tiene una ETP de 1705 mm, según Hargreaves, siendo las estaciones La Mesa y La Esperanza en las cuales se estiman valores menores, 1157 y 1336 mm/año, respectivamente; en las estaciones Pespire, Nacaome y Choluteca se estiman mayores valores de ETP, 2065, 1974 y 1893 mm/año, respectivamente.

Si se divide a Honduras en cuencas, no se observan diferencias significativas entre el promedio de las estaciones que pertenecen a la vertiente del Atlántico (1710 mm/año) y el promedio de las estaciones que pertenecen al Pacífico (1690 mm/año). Podría inferirse que las cuencas del Pacífico tienen menor ETP anual, no obstante, esta deducción no es cierta, debido a que es en las cuencas del Pacífico donde se encuentran las mayores dispersiones (195 mm/año) debido a que en esta zona se encuentran las estaciones con menores ETP (La Esperanza) y con mayores ETP (Pespire, Nacaome, Choluteca). 
Gráfico 5. Relación de la ETP anual, según Hargreaves, y la altitud (msnm)
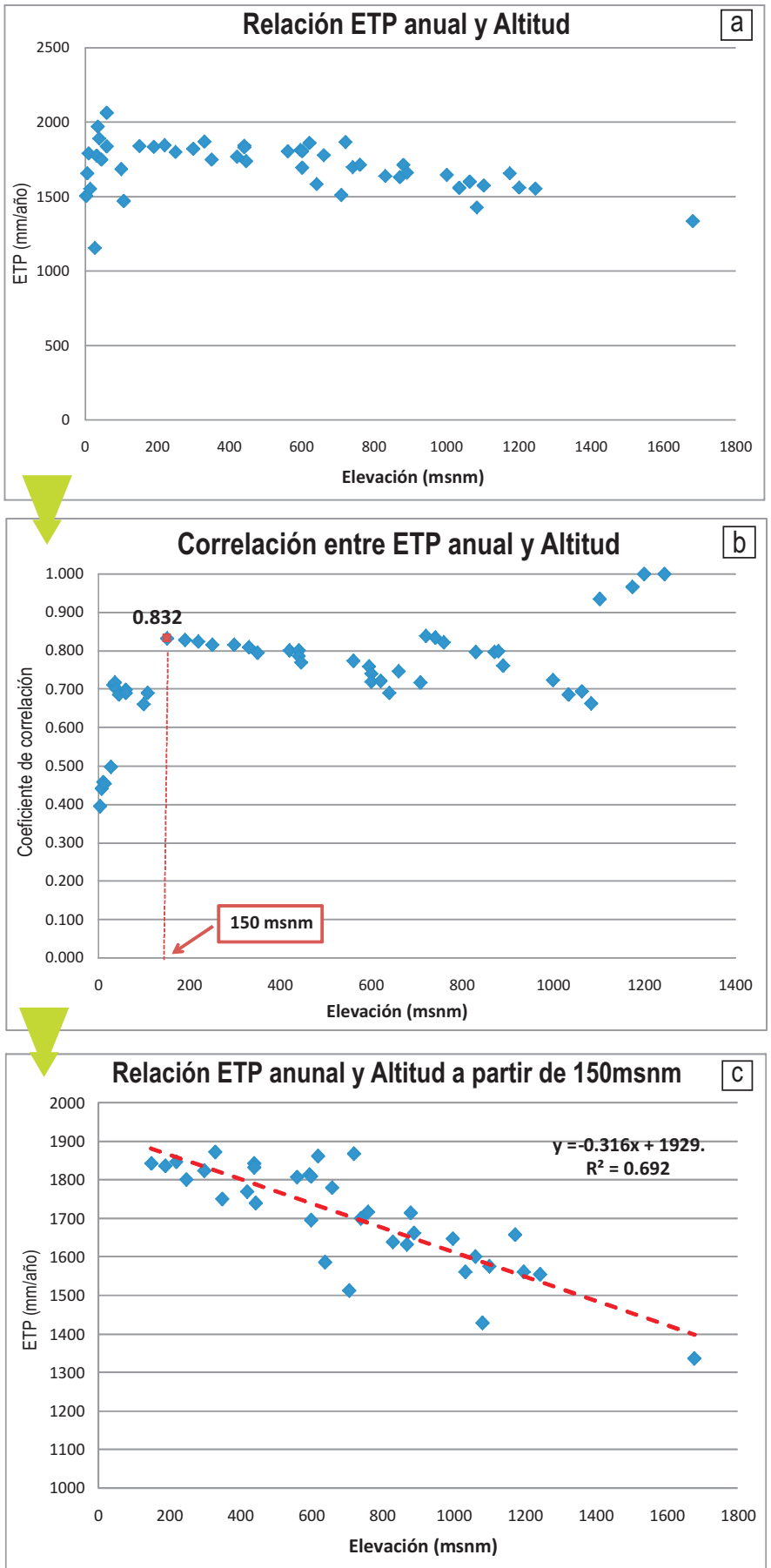
Se observó que la ETP anual tiene una relación inversamente proporcional a la elevación. Tal como se aprecia en el gráfico 5, existe una gran dispersión entre los datos de la ETP y su respectiva elevación (gráfico 5a); sin embargo, al obtener el coeficiente de correlación entre las dos series (gráfico $5 \mathrm{~b}$ ) se deduce que la ecuación de la ETP de Hargreaves tiene buena correlación con la elevación $(r=0.832)$, si se toman sólo los valores con elevaciones mayores a $150 \mathrm{msnm}$ (gráfico 5c).

\section{Comparación de la ETP en Honduras con el mapa Global-PET}

Para la comparación entre los valores estimados a partir de la medición de datos y los valores del mapa Global-PET, se ha realizado un análisis en los puntos específicos del mapa donde se ubica cada estación climática. Se verificaron los datos anuales y mensuales para todas las estaciones escogidas.

En el gráfico 6 se muestra una comparación visual de los diferentes valores en las estaciones, destacándose los datos que arrojan Marale, La Mesa y Santa Rosa de Copán, ya que son las estaciones que presentan las mayores diferencias a lo largo de todo el año. Septiembre es el mes con las mayores diferencias en todas las estaciones y los meses que más coinciden ambas series son abril y mayo.

Gráfico 6. Comparación visual de los valores de la ETP de Honduras y del mapa Global-PET
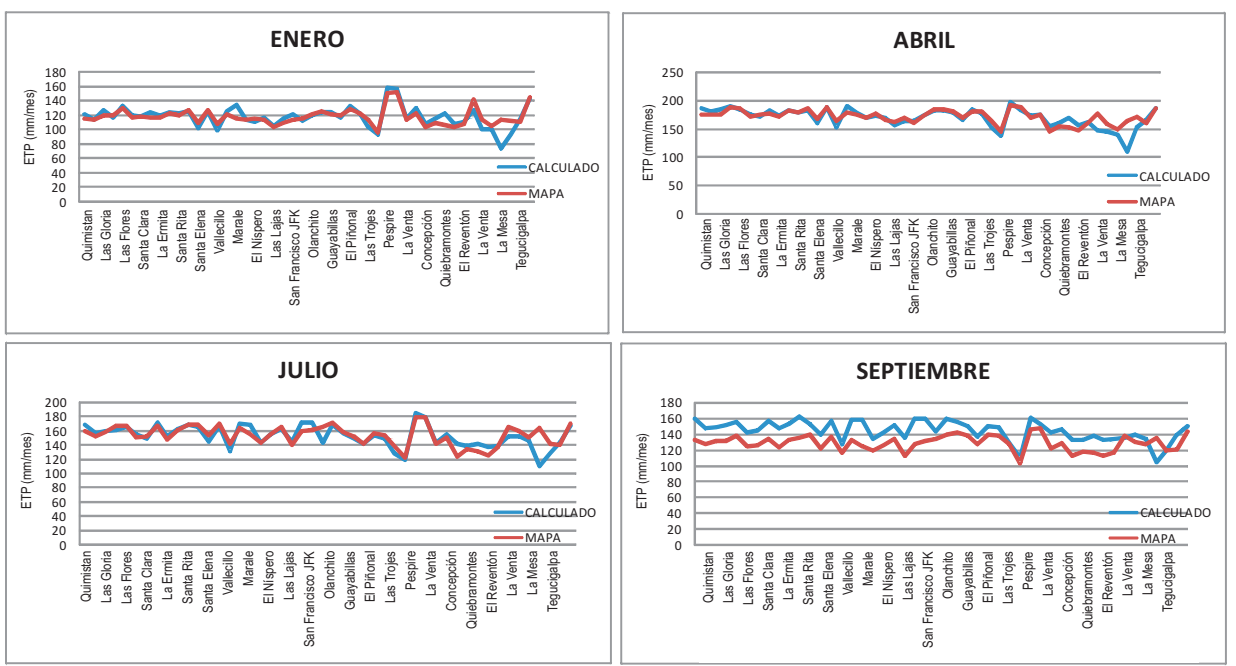
En el gráfico 7 se muestra la relación entre los valores obtenidos de las mediciones y del mapa Global-PET en cada estación. Además, se presentan las ecuaciones de regresión y el valor de $\mathrm{R}^{2}$ para los meses de enero, abril, julio y septiembre y la comparación de los valores anuales.

Gráfico 7. Comparación entre los valores estimados de la ETP-Honduras y los valores del mapa Global-PET
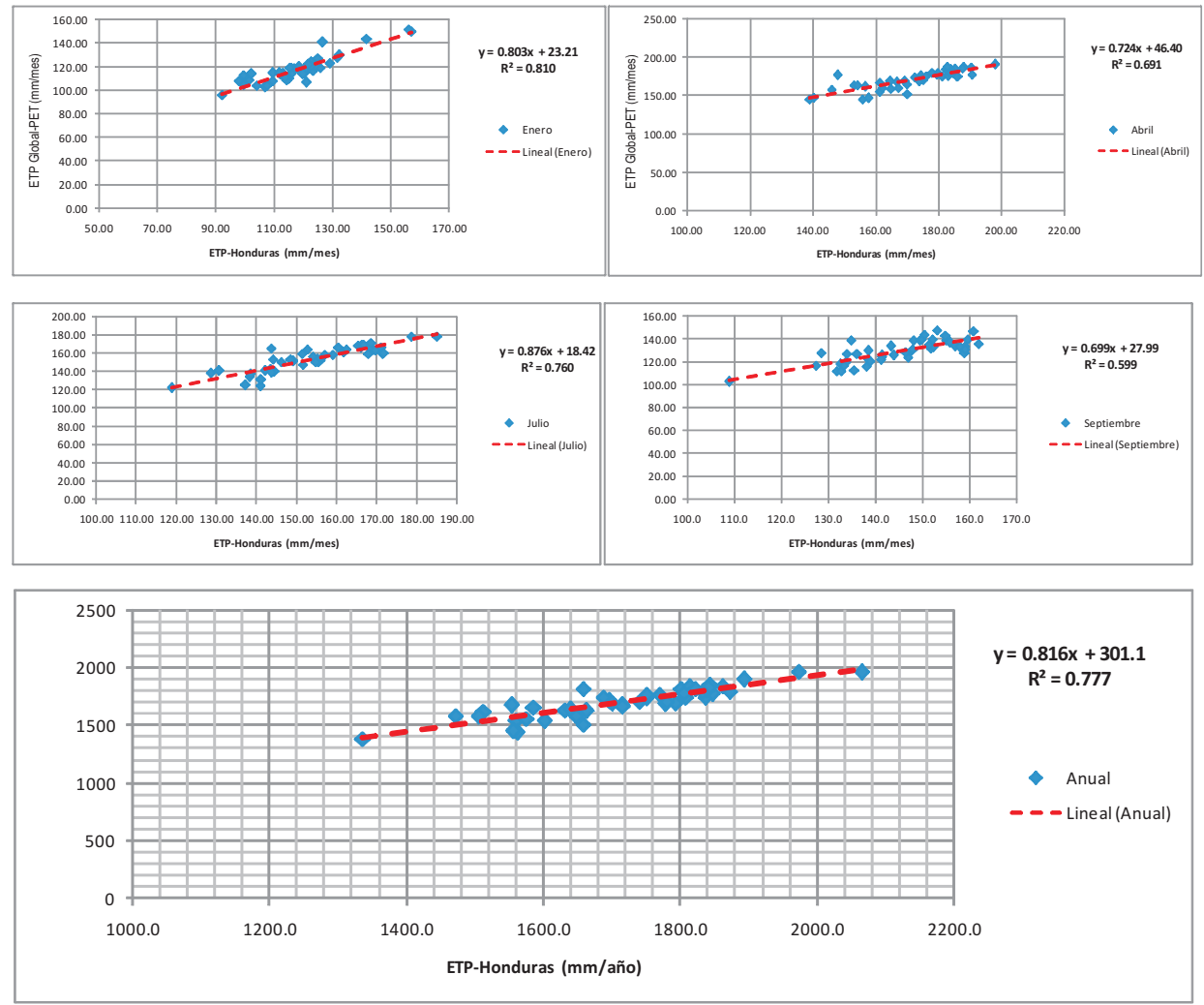

El cuadro 4 presenta los valores estadísticos de primer orden que caracteriza ambas series. Se puede observar que tanto las medias como las desviaciones estándar son muy similares. La mayor varianza en los datos estimados de la ETP se presenta en marzo, abril y mayo; mientras que en los valores obtenidos del mapa se observan en mayo, junio, julio y agosto. Septiembre es el mes que mayor diferencia presenta en la comparación de las medias $(14.6 \mathrm{~mm})$, entretanto el resto de los meses mantiene una diferencia en el promedio de $\pm 1 \mathrm{~mm} / \mathrm{mes}$. 
Cuadro 4. Tabla comparativa de los valores estadísticos de primer orden

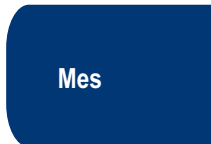

\begin{tabular}{c|c}
$\begin{array}{c}\text { Media-ETP } \\
\text { medida } \\
(\mathrm{mm})\end{array}$ & $\begin{array}{c}\text { Media- } \\
\text { Global-ETP } \\
(\mathrm{mm})\end{array}$ \\
\end{tabular}

Desviación
estándar-
ETP medida
$(\mathrm{mm})$
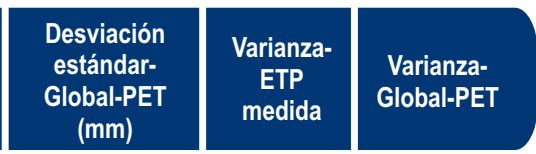

Enero
Febrero
Marzo

116.9

124.6

14.9

11.3

220.9

$164.9 \quad 166.0$

14.3

11.3
10.7
12.0

204.9

16.9

12.0

285.7

Abril

170.5

170.9

16.5

172.4

16.7

1

12.0

13.8

273.7

279.7

12.6

15.5

153.1

152.6

153.1

Agosto

Septiembre

155.1

143.7

154.1

129.1

14.9

13.8

206.4

240.3

12.2

\begin{tabular}{|r|}
\hline 10.0 \\
\hline 10.0 \\
\hline 10.8
\end{tabular}

13.0

Octubre

Noviembre

131.3

Diciembre

111.3

129.1

111.4

109.0

109.2

13.1

13.6

221.2

169.9

10.8

172.7

184.0

11.9

14.5
166.5

128.0

210.6

Anual

1704.6

1702.6

Gráfico 8. Resultados de los test t- student y ANOVA

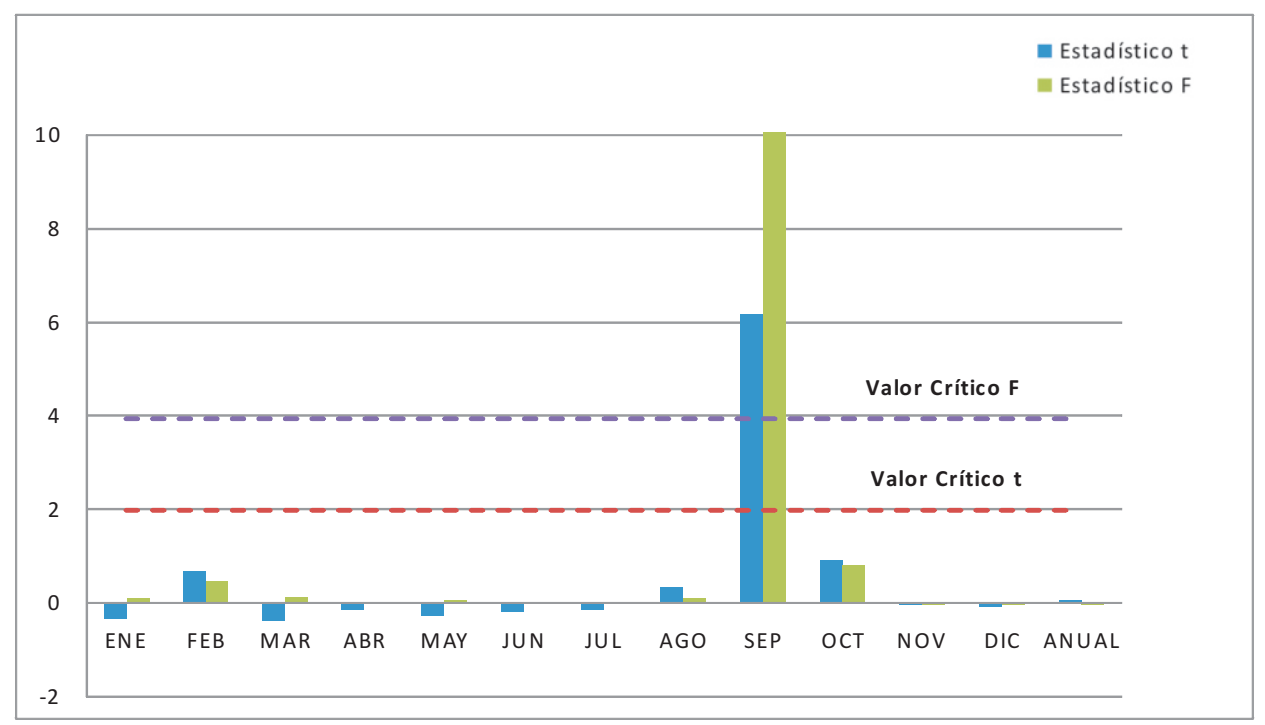

Para probar la hipótesis de que no existe diferencia estadísticamente significativa entre los valores de la ETP estimados y el mapa Global-PET, se ha realizado la prueba de t-student a cada mes y a los datos anuales. Los resultados demuestran que no existen diferencias significativas entre ambas series de datos, excepto en el mes de septiembre. 
También se realizó el testANOVA que determina si existen diferencias significativas en las varianzas de las poblaciones (ver gráfico 8). Al igual que la media, las varianzas (y por tanto sus desviaciones típicas) tampoco mostraron diferencias significativas, excepto en el mes de septiembre.

Finalmente, para validar la relación existente entre ambas series de datos y determinar su nivel confianza, se han obtenido el coeficiente de correlación, el error cuadrático medio y se ha realizado la prueba de Chi cuadrado. En la inspección visual, las estaciones La Mesa, Marale y Santa Rosa de Copán presentaron las mayores diferencias entre los valores estimados y los del mapa Global-PET, por lo que se decidió eliminar estas estaciones del análisis.

El cuadro 5 muestra los resultados de los análisis estadísticos con todas las estaciones, mientras que en el cuadro 6 se muestran los resultados finalmente adoptados en la validación, dejando para futuras investigaciones el estudio en profundidad de las regiones donde se encuentran las estaciones separadas del análisis.

Cuadro 5. Todas las estaciones: coeficiente de correlación, error cuadrático medio (RMSE) y test de chi cuadrado

\begin{tabular}{|l|c|c|c|c|c|}
\hline \multicolumn{1}{|c|}{ Mes } & $\begin{array}{c}\text { Coeficiente } \\
\text { de correlación (r) }\end{array}$ & $\mathbf{R}^{2}$ & RMSE (mm) & RMSE $^{\mathbf{1}} \mathbf{( \% )}$ & $X^{2}$ \\
\hline Enero & 0.8020 & 0.6432 & 8.8 & 7.6 & 0.942 \\
\hline Febrero & 0.7799 & 0.6082 & 9.0 & 7.2 & 0.948 \\
\hline Marzo & 0.7730 & 0.5975 & 10.7 & 6.5 & 0.910 \\
\hline Abril & 0.7405 & 0.5483 & 11.0 & 6.5 & 0.895 \\
\hline Mayo & 0.7507 & 0.5635 & 11.0 & 6.4 & 0.916 \\
\hline Junio & 0.6963 & 0.4849 & 10.5 & 7.0 & 0.924 \\
\hline Julio & 0.7386 & 0.5456 & 10.6 & 7.0 & 0.908 \\
\hline Agosto & 0.7080 & 0.5013 & 10.6 & 6.8 & 0.923 \\
\hline Septiembre & 0.6228 & 0.3879 & 17.9 & 12.4 & 0.000 \\
\hline Octubre & 0.7361 & 0.5419 & 9.0 & 6.9 & 0.974 \\
\hline Noviembre & 0.7983 & 0.6373 & 8.1 & 7.3 & 0.985 \\
\hline Diciembre & 0.8139 & 0.6624 & 8.3 & 7.7 & 0.960 \\
\hline Anual & 0.7347 & 0.5398 & 112.0 & 6.6 & 0.888 \\
\hline
\end{tabular}

${ }^{1}$ Porcentaje estimado en base a la media de la ETP de Honduras. 
Cuadro 6. Coeficiente de correlación, error cuadrático medio y test de chi cuadrado sin las estaciones descartadas

\begin{tabular}{|l|c|c|c|c|c|}
\hline \multicolumn{1}{|c|}{ Mes } & $\begin{array}{c}\text { Coeficiente } \\
\text { de correlación (r) }\end{array}$ & $\mathbf{R}^{2}$ & RMSE (mm) & RMSE $(\%)$ & $X^{2}$ \\
\hline Enero & 0.9004 & 0.8107 & 5.4 & 4.7 & 1.000 \\
\hline Febrero & 0.8709 & 0.7584 & 6.4 & 5.0 & 1.000 \\
\hline Marzo & 0.8539 & 0.7292 & 7.2 & 4.4 & 1.000 \\
\hline Abril & 0.8317 & 0.6917 & 7.6 & 4.5 & 1.000 \\
\hline Mayo & 0.8901 & 0.7923 & 6.6 & 3.8 & 1.000 \\
\hline Junio & 0.8772 & 0.7695 & 6.1 & 4.1 & 1.000 \\
\hline Julio & 0.8720 & 0.7603 & 6.8 & 4.5 & 1.000 \\
\hline Agosto & 0.8667 & 0.7511 & 6.6 & 4.3 & 1.000 \\
\hline Septiembre & 0.7742 & 0.5994 & 16.6 & 11.6 & 0.000 \\
\hline Octubre & 0.9030 & 0.8154 & 5.6 & 4.3 & 1.000 \\
\hline Noviembre & 0.9110 & 0.8299 & 4.8 & 4.4 & 1.000 \\
\hline Diciembre & 0.9192 & 0.845 & 4.9 & 4.5 & 1.000 \\
\hline Anual & 0.8816 & 0.7773 & 66.2 & 3.9 & 1.000 \\
\hline
\end{tabular}

Porcentaje estimado en base a la media de la ETP de Hunduras.

Comparando los valores de los cuadros 5 y 6 se aprecia que todos los meses, excepto septiembre, tienen la máxima valoración en el test de $X^{2}$, existe un incremento significativo en el coeficiente de correlación y una disminución importante en el error cuadrático medio.

Los resultados del cuadro 6 apoyan la hipótesis de que el mapa representa significativamente las estimaciones de la ETP mediante el método de Hargreaves en Honduras; sin embargo, existe una valoración muy pobre en la correlación, el RMSE y el valor de $X^{2}$ para el mes de septiembre, lo que lleva a la conclusión de que este mes no puede ser validado.

\section{CONCLUSIONES}

1. El mapa Global-PET representa adecuadamente las estimaciones de la evapotranspiración potencial (ETP) mensual y anual calculadas, excepto para el mes de septiembre, por el método de Hargreaves en todas las estaciones del país analizadas, con un error medio de $\pm 6.2 \mathrm{~mm}$ a nivel mensual y $\pm 66.2 \mathrm{~mm}$ a nivel anual, lo que equivale a un $88.1 \%$ de confianza.

2. La muestra obtenida del mapa Global-PET, cotejada con las estaciones de Honduras, demuestra que la interpolación utilizada para obtener el mapa es válida para todo el territorio hondureño, lo que significa que los mapas Global- 
PET a nivel mensual (excepto septiembre) y anual puede ser utilizados para determinar laETP media en cualquier punto de Honduras.

3. Existe un gradiente (único, lineal e inversamente proporcional) entre la ETP estimada por el método de Hargreaves y las elevaciones mayores a $150 \mathrm{msnm}$. A elevaciones menores existe una dispersión muy alta de los valores de la ETP, lo que sugiere que intervienen otros procesos en la ETP como, por ejemplo, la humedad relativa y la velocidad del viento, no considerados en la estimación de Hargrevaes. Se puede inferir de las ecuaciones encontradas, que la ETP anual disminuye $32 \mathrm{~mm}$ por cada 100 metros de elevación a partir de los $150 \mathrm{msnm}$, con un $83.2 \%$ de confianza.

4. De acuerdo a las estimaciones utilizando el método de Hargreaves, la ETP media anual en Honduras es de 1705 mm, con sus máximos en abril y mayo; y sus mínimos en noviembre, diciembre y enero. Las estaciones La Mesa, Marale y Santa Rosa de Copán, todas pertenecientes al Servicio Meteorológico Nacional, presentaron las mayores diferencias con los mapas GlobalPET. La Mesa fue la estación con menor ETP del país, más bajo que la estación La Esperanza, el sitio con registros más fríos de Honduras. Los valores de La Mesa no corresponden con su ubicación geográfica, misma situación que Marale y Santa Rosa de Copán, por lo que se debe realizar un estudio más detallado alrededor de estas estaciones para verificar la validez de esta estimación.

5. Aunque el esfuerzo por medir datos climáticos es valioso, la sola medición no garantiza que el dato obtenido pueda utilizarse en estudios posteriores. Cuarenta y ocho (48) estaciones meteorológicas del país que miden temperatura han servido de base a este estudio de 79 disponibles y de estas 48 estaciones el dato de temperatura media mensual no fue consistente, por lo que se descartó y se estimó a partir de otros parámetros. Las autoridades competentes deben analizar una forma de evaluación y homogenización institucional de los registros de las estaciones.

6. Los mapas mensuales, excepto septiembre, y anual de Global-PET representan una nueva base de datos validada a nivel nacional, sin costo alguno y accesible desde internet para quien quiera hacer uso de ella. Para estimar la ETP diaria o semanal requerida, solo basta identificar la zona de interés, obtener el dato mensual de la ETP y dividirlo entre el número de semanas 0 días, según sea el caso. 


\section{AGRADECIMIENTOS}

Al proyecto de actualización del Balance Hídrico de Honduras de la Secretaría de Recursos Naturales y Ambiente (SERNA) y al Instituto Hondureño de Ciencias de la Tierra de la Universidad Nacional Autónoma de Honduras (IHCT-UNAH), por el apoyo brindado a esta investigación.

\section{BIBLIOGRAFÍA}

Allen, R.G.; Pereira, L.S.; Raes, D. \& Smith, M. (1998). Crop evapotranspiration: Guidelines for computing crop requirements. Irrigation and Drainage, 56. Rome. CEDEX. (2013). Cálculos hidrometeorológicos de aportaciones y crecidas. Manual CHAC.

Comisión Nacional de Riego. (1997). Cálculo y cartografía de la evapotranspiración potencial en Chile. Santiago: Comisión Nacional de Riego.

Droogers, P. and Allen, R.G. (2002). Estimating reference evapotranspiration under inaccurate data conditions. Irrigation and Drainage Systems, (16)1, 33-45. Rome.

Hargreaves, G. H. (1994). Defining and using reference evapotranspiration. Journal of Irrigation and Drainage Engineering, (120)6, 1132-1139.

Hargreaves, G.H. \& Allen, R.G. (2003). History and evaluation of Hargreaves evapotranspiration equation. Journal of Irrigation and Drainage Engineering, (129)1, 53-63.

Hargreaves, G.L.; Hargreaves, G.H. y Riley, J.P. (1985). Agricultural benefits for Senegal River Basin. Journal of Irrigation and Drainage Engineering, 111, 113124.

Hijmans, R.J.; Cameron, S.E.; Parra, J.L.; Jones, P.G. \& Jarvis, A. (2004). The World Clim interpolated global terrestrial climate surfaces, version 1.3. Recuperated of: http://biogeo.berkeley.edu/.

Jiménez, F. y Hernández, R. (1990). Estudio de la evapotranspiración potencial en

Honduras. Centro Agronómico Tropical de Investigación y Enseñanza (CATIE), Programa Manejo Integrado de los Recursos Naturales, Proyecto Regional de Agrometeorología.

López Moreno, J. L.; Hess, T. M. and White, M. (2009). Estimation of reference evapotranspiration in a mountainous mediterranean site using the PenmanMonteith equation with limited meteorological Data. Pirineos, 164, 7-31.

Mckenney, M.S. and Rosenberg, N. J. (1993). Sensitivity of some potential evapotranspiration estimation methods to climate change. Agricultural and Forest Meteorology, (64), 81-110.

Menne, M.J.; Williams, C. N.; Vose, R. S. (2009). The U.S. Historical Climatology 
Network Monthly Temperature Data, Version 2. American Meteorological Society, 993-1007.

Sánchez, M. I. (1992). Métodos para el estudio de la evaporación y evapotranspiración. Logroño: Geoforma Ediciones.

SERNA/CEDEX. (2002). Balance Hídrico de Honduras. Agencia Española de Cooperación Internacional.

Skolov, A.A. y Chapman, T.G. (1981). Métodos de cálculo del balance hídrico. Guía internacional de investigación y métodos. Instituto de Hidrología de España, UNESCO.

Temesgen, B.; Eching, S.; Davidoff, B. and Frame, K. (2005). Comparison of some reference evapotranspiration equations for California. Journal of Irrigation and Drainage Engineering, 73-84.

Thornthwaite, C.W. (1948). An approach toward a rational classification of climate. Geograph. Rev., 38, 55.

Trabucco, A., and Zomer, R.J. (2009). Global Aridity Index (Global-Aridity) and Global Potential Evapo-Transpiration (Global-PET) Geospatial Database. CGIAR Consortium for Spatial Information. Recuperated of: http://www.csi.cgiar.org

Valenzuela, A. y Ferreira, V. (1985). Variación de la evapotranspiración potencial en Chile. Agro-Ciencia, (1)1, 15-21.

Xu, C. Y. and Singh, V. P. (2001). Evaluation and generalization of temperaturebased methods for calculating evaporation. Hydrological Processes, 13, 305319.

Zúniga, E. (1990). Las modalidades de la lluvia en Honduras. Tegucigalpa: Editorial Guaymuras. 